\title{
HÁBITOS SALUDABLES Y MÉTODOS PARA ALCANZAR UN ESTADO EMOCIONAL ÓPTIMO EN LA MENOPAUSIA ${ }^{1}$
}

\author{
María del Mar Crisol-Martínez ${ }^{2}$
}

\begin{abstract}
Hábitos saludables y métodos para alcanzar un estado emocional óptimo en la menopausia Resumen: Existen dentro de la vida de una mujer, etapas reproductivas y no reproductivas. Dentro de las no reproductivas, nos encontramos con la menopausia, que generalmente coincide con la mediana edad, alrededor de los 50 años. Durante la perimenopausia, que es la etapa que rodea a la menopausia, la mujer es más vulnerable a sufrir depresión. Esto puede coincidir con otros factores estresantes en esta etapa, como pueden ser cuando los hijos dejan el hogar por primera vez, para estudiar o a trabajar, lo que se conoce por el "síndrome de nido vacío". La aparición de síntomas menopáusicos como pueden ser los sofocos, el insomnio, o el cansancio, repercute en el estado de humor de la mujer, es decir, en su estado emocional. A lo largo de este periodo se producen cambios físicos, aunque también suelen ocurrir cambios psicológicos, como puede ser llanto fácil, ansiedad, etc. Es por ello necesario pensar en una estrategia que sirva de profilaxis o de prepa- ración a la llegada de esta etapa. El coaching es una herramienta que, partiendo de las necesidades planteadas por cada mujer, puede ayudarla a alcanzar sus objetivos. La estrategia de coaching, puede ayudar a la mujer a sentirse más motivada, más segura de sí misma y, por tanto, puede reducir la aparición de una sintomatología depresiva. El coaching puede ayudar a la mujer a alcanzar su bienestar emocional, con el objetivo de vivir una madurez plena.
\end{abstract}

Palabras clave: menopausia, ansiedad, depresión, estado de ánimo, estado emocional, coaching.

\section{Healthy Habits to Achieve an Optimal Emotional State during Menopause}

Abstract: During a woman's life, there are reproductive and non-reproductive stages. Among the non-reproductive, the menopause stage, which generally occurs during middle age, around the age of 50. During the perimenopause, a woman is more vulnerable to suffer depression. This may coincide with other stress-promoting factors, such as the "empty nest syndrome" (i.e. when children leave home for the first time). The appearance of menopausal symptoms such as hot flushes, insomnia or fatigue, has an impact on her emotional state. A number of physical changes occur over this period, sometimes coupled with psychological changes such as anxiety and high emotional sensitivity. Thus, a strategy to cope with these changes can be highly beneficial. In this sense, coaching is a tool which can help a woman to reach her personal goals, based on her individual needs. It can help a woman to feel more motivated and more self-confident and it lowers the risk of depression. Coaching can help women to reach emotional well-being while enjoying full maturity.

Keywords: menopause, anxiety, depression, mood, emotional state, coaching.

\section{Introducción}

A lo largo de la vida de una mujer, existen distintas etapas reproductivas y no

\footnotetext{
1 Fecha de recepción: 09/12/2018.

Fecha de aceptación: 30/12/2019.

2 Técnica SICA/Coach especializada en mujeres, Universidad de Almería, España; $\square$ mmarcrisol@ hotmail.com.
} 
reproductivas. Al conjunto de todas las etapas se denomina climaterio. Dentro de éste se encuentran la premenopausia, perimenopausia, menopausia y la postmenopausia. La primera etapa reproductiva ocurre en el transcurso de la niñez a la adolescencia, es decir, cuando aparece la primera menstruación. Se producen una serie de cambios físicos, orgánicos y generalmente, psicológicos. A partir de este momento, el organismo de la mujer se encuentra preparado para ser madre. En los países desarrollados, la edad en que la mujer decide tener hijos es cada vez más tardía. Conseguir un empleo y mantener una estabilidad laboral son tareas arduas y complicadas. Esto puede hacer que la mujer se cuestione la maternidad. En algunos casos, estas dudas conllevan una falta de plenitud en la mujer, por el hecho de no poder ser madre. La edad fértil es el inconveniente principal de la mujer, ya que este periodo biológico tiene un límite, que frecuentemente coincide con el momento en que la mujer ha conseguido la estabilidad laboral. Una vez alcanzada, algunas mujeres se plantean ser madres.

\section{Climaterio}

La etapa que precede a la menopausia es la premenopausia. Investigadores definen que la transición a la menopausia es el período comprendido entre los períodos poco frecuentes e irregulares hasta la menstruación final que marca el inicio del período posmenopáusico (Taylor et al., 2014). En algunos estudios se sugiere que la mujer está en el inicio a la transición de la menopausia, cuando existe un cambio en el intervalo menstrual de al menos 7 días, entre ciclo y ciclo, o bien, se ha omitido algún período 
menstrual (Butler et al., 2011). Autores sugieren que la premenopausia está dentro de la menstruación regular (Hunter et al., 2007).

La menopausia es la etapa en la que la mujer deja de ser fértil. Se define como la etapa que comienza 12 meses después del último período menstrual. Es un cambio natural, caracterizado por la disminución de los niveles de estrógenos, representando el paso de la edad fértil a la vejez. La Organización Mundial de la Salud (OMS), confirma que el período de edad en la que puede aparecer la menopausia está entre los 48 y 52 años, como consecuencia de la pérdida de actividad en los folículos ováricos, seguida de un cese de la hemorragia menstrual durante al menos un año (OMS, 1996). Una vez que ocurren los 12 meses seguidos sin menstruación es cuando existe la menopausia confirmada. Algunos autores nos informan que una mujer puede estar en la etapa de la perimenopausia si estando en una edad igual o mayor de 45 años, presenta cambios en el ciclo menstrual y síntomas que puedan identificar la llegada de la menopausia (Hale et al., 2014), como la disminución apreciable en la du- ración del ciclo. En definitiva, se considera que la perimenopausia incluye la fase inmediatamente anterior a la menopausia y el primer año después de la menopausia y se caracteriza por los cambios en la regularidad de los ciclos menstruales durante los 12 meses anteriores.

La postmenopausia comprende el intervalo de 1 a 6 años tras la menopausia. Existen mujeres que se han sometido a histerectomía o que están bajo tratamiento hormonal y es difícil clasificarlas dentro de estas etapas y por lo general, se clasifican por separado. 


\section{Sintomatología en la menopausia:}

Dentro del climaterio se producen una serie de molestias que sufre la mujer, debido en parte a las fluctuaciones hormonales, endocrinas, físicas y psíquicas, que se van produciendo durante esta fase. Butler et al., (2011) indican que la transición a la menopausia parece estar asociada con el aumento de los síntomas asociados a esta etapa, como son los sofocos, el estado de ánimo negativo, falta de sueño o insomnio y síntomas urogenitales. Existe sintomatología vasomotora (como son los sudores nocturnos, los sofocos) sintomatología cognitiva (aumento de la confusión y del olvido) así como cambios somáticos (dolores de cabeza) (Gibbs et al., 2013). Hunter (2007) añade la posibilidad de pérdida de la libido, así como la aparición de ansiedad o depresión. En algunos casos, la mujer presenta irritabilidad, falta de memoria y pérdida de concentración, cambios en el humor, cansancio, dolor de extremidades, pérdida de energía, así como la presencia de la piel seca.

De todos los síntomas psicológicos, el bajo estado de ánimo que puede presentar la mujer puede ir asociado a la desregulación hormonal, como consecuencia de la ausencia de la menstruación. En este sentido, Richard et al. (2014) argumentan que las mujeres son más propensas a padecer depresión, y una de las hipótesis está ligada a los cambios en los niveles de ciertas hormonas sexuales, como son los estrógenos, que influyen sobre dicha patología. De hecho, la disminución de estrógenos puede estar vinculada a la aparición de trastornos depresivos, así como una disminución de las funciones cognitivas (Weber et al., 2014). Este mismo estudio indica que existe un mayor riesgo de sufrir de- presión 
durante las etapas en donde ocurren cambios hormonales, particularmente durante la perimenopausia, y la postmenopausia. De hecho, las mujeres en estas etapas son más vulnerables a padecer sintomatología depresiva (Almeida et al., 2016). Grochans et al. (2013) indican que el 90\% de las mujeres sufren estos trastornos en el estado anímico, repercutiendo en su salud mental y manifestándose con cambios de humor repentino, fatiga, nerviosismo, estrés, así como sintomatología depresiva.

Adicionalmente, la transición de etapas durante la madurez puede coincidir con circunstancias no favorables que le rodean y que pueden ser responsables de que aparezcan síntomas psicológicos (Pimenta et al., 2012). Una de las circunstancias más comunes que ocurren durante la edad mediana de la mujer es el "síndrome del nido vacío", cuando los hijos salen del hogar por primera vez para estudiar o a trabajar, lo que puede repercutir negativamente, apareciendo síntomas como ansiedad o depresión. Hay una serie de circunstancias familiares cada vez más frecuentes, que pueden ahondar la vulnerabilidad emocional femenina. Uno de ellos puede ser la ruptura de la pareja, que suele ir unida a un alto estrés emocional. En esta etapa de la vida, es también común vivir el envejecimiento y consecuentemente, la aparición de enfermedades en los padres u otros familiares. La mujer ha dejado de criar a sus hijos, pero ahora tiene a su cargo a los padres al hacerse mayores y/o enfermar.

Otras circunstancias relativamente comunes en la menopausia son problemas de salud, o ciertas dificultades económicas, que pueden minar el estado de ánimo de la mujer. Todas las circunstancias anteriormente mencionadas suponen obstáculos que pueden perjudicar la salud mental. Pimenta et al., (2012) señalan que la aparición de los síntomas en la menopausia puede estar influenciados por otros factores como es la categoría profesional, la educación, así como el tabaquismo y el estilo de vida. Además, identifican 
que algunos eventos negativos durante la vida de la mujer pueden llegar a ser predictores de la sintomatología en el climaterio.

\section{Depresión}

La OMS nos dice cómo la depresión afecta a la salud de las personas, es decir, en su calidad de vida (OMS, 2001 citados por Torres et al., 2012). Es una de las principales causas de discapacidad a nivel mundial y una enfermedad mental muy común en nuestra sociedad (Ginty et al., 2015). El paciente se caracteriza por un estado de ánimo muy bajo, sumido en una profunda tristeza, pérdida de interés por realizar actividades placenteras, baja autoestima, pérdida de apetito, insomnio, cansancio y dificultad para concentrarse (Richard et al. 2014). La depresión es el resultado de la interacción de numerosos factores como pueden ser genéticos, psicológicos, y ambientales. Dentro de esta patología también hay que tener en cuenta los antecedentes familiares, la economía familiar del paciente, la educación recibida, así como otros factores que pueden tener relación con la aparición de esta sintomatología depresiva.

Richard et al., (2014) subrayan que la depresión es uno de los problemas más importantes de la salud pública, siendo una de las causas más frecuentes de baja laboral, seguida de la ansiedad. Existe un $20 \%$ de personas en el mundo que han sido diagnosticadas de depresión mayor (Yu et al., 2013).

Niu et al., (2013) sugieren que la carga de morbilidad de enfermedades asociadas al estrés, como es la sintomatología depresiva y el trastorno de ansiedad, ha tenido un incremento muy rápido en estos últimos años. 


\section{Tratamiento y hábitos saludables en la depresión}

Como tratamiento farmacológico se encuentran los fármacos antidepresivos y los ansiolíticos, aunque la labor de muchos investigadores es encontrar terapias farmacológicas con menos efectos secundarios, así como otras estrategias terapéuticas selectivas que puedan abordar este trastorno (Wang et al., 2012).

Algunos estudios señalan que el climaterio no es una patología, aunque puede llegar a necesitar terapias específicas dependiendo de la sintomatología que presente (PérezRoncero et al., 2013). De forma general, un cambio en el estilo de vida sería beneficioso para la depresión y podría contribuir como un tratamiento coadyuvante, actuando como un factor de prevención para la depresión (Serrano Ripoll et al., 2015). Por ello, es importante tomar medidas preventivas asociadas a una mejora del estilo de vida, como, por ejemplo, un cambio en la dieta. Además, es importante incluir, bajo un punto de visto psicológico, un servicio de apoyo para tratar de manejar el estrés, acompañado de técnicas de relajación.

Según la OMS, mantener un buen estado de ánimo y mantener un nivel óptimo de estrés es fundamental para nuestra salud y bienestar. Si una mujer se encuentra bien emocionalmente va a estar más capacitada para hacer frente a su día a día y a cualquier circunstancia que pueda aparecer. Debido a que la menopausia es una de las etapas más 
vulnerables a la sintomatología depresiva y dado que existen estudios que lo evidencian, se podría conseguir que las mujeres en una edad comprendida entre 45-55 años, tuvieran unas pautas que les ayudara a sentirse bien emocionalmente.

\section{Coaching como herramienta durante la menopausia}

El coaching es una herramienta que ayuda a una persona a lograr las metas que se proponga. Es un método en el que el coach acompaña al cliente, durante un proceso que suele constar de varias sesiones. En cada sesión, se utilizan una serie de preguntas que va haciendo el coach a su cliente. Estas preguntas están orientadas a conseguir que sea el cliente el que piense, reflexione, se escuche y realice un plan de acción para llevar a cabo su propósito. Por tanto, la responsabilidad de conseguir el objetivo es del cliente. Cuando consigue el objetivo, generalmente se siente con un grado de satis- facción alto por haberlo hecho él solo.

Existen evidencias que demuestran la eficacia del coaching en la mejora física y emocional de mujeres en distintas etapas del climaterio. Por ejemplo, Fujimoto et al., (2017) demostraron la eficacia del coaching para mejorar la salud de mujeres japonesas de 40 a 60 años menopáusicas, que no recibían terapia hormonal. El grupo de intervención de mujeres recibió tres sesiones de coaching al mes durante 3 meses, las cuales mejoraron la salud de las mujeres menopáusicas. Otro estudio demostró que una intervención de coaching durante la menopausia mejoró los resultados relacionados con la salud mental (Almeida et al., 2016). Por tanto, el coaching podría desempeñar un papel muy importante 
en la reducción de síntomas depresivos en la transición a la menopausia.

\section{Conclusión}

Con todo lo anteriormente mencionado, estos métodos y pautas podrían usarse como profilaxis para el bienestar de las mujeres de mediana edad. La integración de varios de estos métodos podría evitar síntomas de ansiedad, así como la aparición de depresión en la mujer de mediana edad. De esta manera, se podría reducir el consumo de fármacos antidepresivos y ansiolíticos, disminuyendo así el gasto sanitario de este grupo de medicamentos. Así mismo, es probable que una mejora de salud disminuyera las bajas laborales de mujeres asociados a depresión o crisis de ansiedad. Por último, se podría preparar a la mujer para asumir los cambios físicos, orgánicos y psíquicos que pueden conllevar la llegada de la menopausia. En resumen, sería recomendable poner todos estos hábitos y métodos en la práctica, ayudando a las mujeres a que vivan una madurez plena, evitando la llegada de posibles patologías psíquicas, y consiguiendo un óptimo bienestar en las mujeres que así lo necesiten.

\section{Referencias bibliográficas}


Almeida, O. 2016. "Reducing depression during the menopausal transition with health coaching: Results from the healthy menopausal transition randomised controlled trial". Maturitas, 92(0), 41-48

Butler, L., \& Santoro, N. 2011. "The reproductive endocrinology of the menopausal transition”. Steroids, 76 (7): 627-635.

Fujimoto, K. 2017. "Effectiveness of coaching for enhancing the health of menopausal Japanese women". J Women Aging, 29(3): 216-229.

Gibbs, Z., Lee, S., \& Kulkarni, J. 2013. "Factors associated with depression during the perimenopausal transition”. Women's Health Issues, 23(5): e301-e307.

Ginty, A. T., \& Conklin, S. M. (2015). Short-term supplementation of acute long-chain omega-3 polyunsaturated fatty acids may alter depression status and decrease symptomology among young adults with depression: A preliminary randomized and placebo controlled trial. Psychi-atry Research, 229(1-2), 485-489.

Grochans, E., Grzywacz, A., Jurczak, A., Samochowiec, A., Karakiewicz, B., Brodowska, A., ... Samochowiec, J. (2013). The 5HTT and MAO-A polymorphisms associate with depressive mood and climacteric symptoms in postmenopausal women. Progress in Neuro-Psychophar- macology and Biological Psychiatry, 45(0), 125-130.

Hale, G. E., Robertson, D. M., \& Burger, H. G. (2014). The perimenopausal woman: Endocrinology and management. The Journal of Steroid Biochemistry and Molecular Biology, 142(0), 121- 131.

Hunter, M., \& Rendall, M. (2007). Bio-psycho-socio-cultural perspectives on menopause. Best Practice \& Research Clinical Obstetrics \& Gynaecology, 21(2), 261-274.

Niu, K., Guo, H., Kakizaki, M., Cui, Y., Ohmori-Matsuda, K., Guan, L., . . Nagatomi, R. (2013). A tomato-rich diet is related to depressive symptoms among an elderly population aged 70 years and over: A population-based, cross-sectional analysis. Journal of Affective Disor- ders, 144(1-2), 165-170.

Pérez-Roncero, G. R., Martínez-Dearth, R., López-Baena, M. T., \& Ornat-Clemente, L. (2013). Sín- tomas relacionados con el climaterio en mujeres residentes en la provincia de Zaragoza. En-fermería Clínica, 23(6), 252-261.

Pimenta, F., Leal, I., Maroco, J., \& Ramos, C. (2012). Menopausal symptoms: Do life events pre- dict severity of symptoms in peri- and post-menopause? Maturitas, 72(4), 324-331.

Richard, A., Rohrmann, S., Mohler-Kuo, M., Rodgers, S., Moffat, R., Güth, U., \& Eichholzer, M. (2014). Urinary phytoestrogens and depression in perimenopausal US women: NHANES 2005-2008. Journal of Affective Disorders, 156(0), 200-205.

Serrano Ripoll, M. J., Oliván-Blázquez, B., Vicens-Pons, E., Roca, M., Gili, M., Leiva, A., . . . Gar- cía-Toro, M. (2015). Lifestyle change recommendations in major depression: Do they 
work? Journal of Affective Disorders, 183, 221-228.

Torres, S. J., \& Nowson, C. A. (2012). A moderate-sodium DASH-type diet improves mood in postmenopausal women. Nutrition, 28(9), 896-900.

Vivian-Taylor, J., \& Hickey, M. (2014). Menopause and depression: Is there a link? Maturitas, (0)

Wang, Y., Yang, M., Lee, S., Davis, C. G., Kenny, A., Koo, S. I., \& Chun, O. K. (2012). Plasma total antioxidant capacity is associated with dietary intake and plasma level of antioxidants in postmenopausal women. The Journal of Nutritional Biochemistry, 23(12), 1725-1731.

Weber, M. T., Maki, P. M., \& McDermott, M. P. (2014). Cognition and mood in perimenopause: A systematic review and meta-analysis. The Journal of Steroid Biochemistry and Molecular Bi- ology, 142(0), 90-98.

World Health Organization (1996). Research on menopause in the nineties. Report of a scientific group of WHO Geneve: WHO

Yu, Y., Wang, R., Chen, C., Du, X., Ruan, L., Sun, J., . . X Xu, Y. (2013). Antidepressant-like effect of trans-resveratrol in chronic stress model: Behavioral and neurochemical evidences. Journal of Psychiatric Research, 47(3), 315-322. 